\title{
Anatomical Therapeutic Chemical Classification System
}

National Cancer Institute

\section{Source}

National Cancer Institute. Anatomical Therapeutic Chemical Classification System. NCI

Thesaurus. Code C154331.

A World Health Organization (WHO) classification system for medicinal substances where active substances are divided into different groups according to the organ or system on which they act and their therapeutic, pharmacological, and chemical properties. Drugs are classified in groups at five different levels. 